\title{
Stability of hyperbolic space under Ricci flow
}

\author{
Oliver C. Schnürer, Felix Schulze and Miles Simon
}

\begin{abstract}
We study the Ricci flow of initial metrics which are $C^{0}$-perturbations of the hyperbolic metric on $\mathbb{H}^{n}$. If the perturbation is bounded in the $L^{2}$-sense, and small enough in the $C^{0}$-sense, then we show the following: In dimensions four and higher, the scaled Ricci harmonic map heat flow of such a metric converges smoothly, uniformly and exponentially fast in all $C^{k}$-norms and in the $L^{2}$-norm to the hyperbolic metric as time approaches infinity. We also prove a related result for the Ricci flow and for the two-dimensional conformal Ricci flow.
\end{abstract}

\section{Introduction}

We investigate stability of hyperbolic space under Ricci flow

$$
\begin{cases}\frac{\partial}{\partial t} g_{i j}=-2 \operatorname{Ric}(g(t)), & \text { on } \mathbb{H}^{n} \times(0, \infty), \\ g(0)=g_{0}, & \text { on } \mathbb{H}^{n}\end{cases}
$$

As hyperbolic space expands under Ricci flow, it is convenient to consider the following modified Ricci flow:

$$
\begin{cases}\frac{\partial}{\partial t} g_{i j}=-2 \operatorname{Ric}(g(t))-2(n-1) g_{i j}(t), & \text { on } \mathbb{H}^{n} \times(0, \infty), \\ g(0)=g_{0}, & \text { on } \mathbb{H}^{n}\end{cases}
$$

The hyperbolic metric $h$ of sectional curvature -1 is a stationary point to (1.2).

Note that by Lemma A.4, up to rescaling, this flow equation is equivalent to (1.1). As (1.1) and (1.2) are degenerate parabolic equations, we consider the following modified (or rescaled) Ricci harmonic map heat flow which is 
similar to DeTurck flow [3]:

$$
\begin{cases}\frac{\partial}{\partial t} g_{i j}=-2 \operatorname{Ric}(g(t))+\nabla_{i} V_{j}+\nabla_{j} V_{i}-2(n-1) g_{i j}(t), & \text { on } \mathbb{H}^{n} \times(0, \infty), \\ g(0)=g_{0}, & \text { on } \mathbb{H}^{n},\end{cases}
$$

where $V_{i}=g_{i k}\left({ }^{g} \Gamma_{r s}^{k}-{ }^{h} \Gamma_{r s}^{k}\right) g^{r s}$ and $h$ is the hyperbolic metric on $\mathbb{H}^{n}$ of sectional curvature equal to -1 . Once again, up to rescaling, this is equivalent to standard Ricci harmonic map heat flow.

We consider perturbations that are close to hyperbolic space.

Definition 1.1. Let $g$ be a metric on $\mathbb{H}^{n}$. Let $\varepsilon>0$. Then $g$ is $\varepsilon$-close to $h$ if

$$
(1+\varepsilon)^{-1} h \leq g \leq(1+\varepsilon) h
$$

in the sense of matrices.

Let $\left(\lambda_{i}\right)_{1 \leq i \leq n}$ denote the eigenvalues of $\left(g_{i j}\right)$ with respect to $\left(h_{i j}\right)$. Then this is equivalent to $(1+\varepsilon)^{-1} \leq \lambda_{i} \leq 1+\varepsilon$ for $1 \leq i \leq n$.

We denote with $\mathcal{M}^{k}\left(\mathbb{H}^{n}, I\right)$ the space of families $(g(t))_{t \in I}$ of sections in the space of Riemannian metrics on $\mathbb{H}^{n}$ which are $C^{k}$ on $\mathbb{H}^{n} \times I$. Similarly, we define $\mathcal{M}^{\infty}, \mathcal{M}_{\mathrm{loc}}^{k}$ and use $\mathcal{M}^{k}\left(\mathbb{H}^{n}\right)$ if the metric does not depend on $t$. We wish to point out that we use $C^{k}$ on non-compact sets to denote the space, where covariant derivatives with respect to the hyperbolic metric $h$ of order up to $k$ are continuous and in $L^{\infty}$. We also use $C_{\mathrm{loc}}^{k}$. For our convenience, we define $\mathcal{M}_{0}^{\infty}\left(\mathbb{H}^{n},[0, \infty)\right)$ to be the set of all metrics in $\mathcal{M}^{\infty}\left(\mathbb{H}^{n},(0, \infty)\right) \cap$ $M_{\mathrm{loc}}^{\infty}\left(\mathbb{H}^{n},[0, \infty)\right)$ which are smooth for positive times and, when restricted to time intervals of the form $[\delta, \infty), \delta>0$, are uniformly bounded in $C^{k}$ for any $k \in \mathbb{N}$.

We use $|Z| \equiv{ }^{h}|Z|$ to denote the norm of a tensor $Z$ with respect to the hyperbolic metric $h$. Unless stated otherwise, $B_{R}(0)$ denotes a geodesic ball around a fixed point in hyperbolic space which we denote by $0 .\|\cdot\|_{L^{2}}$ denotes the $L^{2}$-Norm with respect to the hyperbolic metric $h$. Sometimes, we write $x \rightarrow \infty$ instead of $|x| \rightarrow \infty$. Moreover, we use the Einstein summation convention and denote generic constants by $c$.

Our main theorem is

Theorem 1.1. Let $n \geq 4$. For all $K>0$ there exists $\varepsilon_{1}=\varepsilon_{1}(n, K)>0$ such that the following holds. Let $g_{0} \in \mathcal{M}^{0}\left(\mathbb{H}^{n}\right)$ satisfy $\int_{\mathbb{H}^{n}}\left|g_{0}-h\right|^{2} d \mathrm{vol}_{h} \leq$ 
$K$ and $\sup _{\mathbb{H}^{n}}\left|g_{0}-h\right| \leq \varepsilon_{1}$. Then there exists a solution $g \in \mathcal{M}_{0}^{\infty}\left(\mathbb{H}^{n},[0, \infty)\right)$ to (1.3) such that

$$
\sup _{\mathbb{H}^{n}}|g(t)-h| \leq C(n, K) \cdot \mathrm{e}^{-\frac{1}{4(n+2)} t}
$$

Moreover, $g(t) \rightarrow h$ exponentially in $C^{k}$ as $t \rightarrow \infty$ for all $k \in \mathbb{N}$.

There might be different solutions to the ones obtained by our construction. The following theorem does not assume that the solution in question comes from the theorem above.

Theorem 1.2. Let $n \geq 4$. For all $K>0$ there exists $\varepsilon_{1}=\varepsilon_{1}(n, K)>0$ such that the following holds. Let $g \in \mathcal{M}_{0}^{\infty}\left(\mathbb{H}^{n} \times[0, \infty)\right)$ be a solution to $(1.3)$ that satisfies $\int_{\mathbb{H}^{n}}|g(0)-h|^{2} d \operatorname{vol}_{h} \leq K, \lim _{r \rightarrow \infty}\|g(0)-h\|_{L^{\infty}\left(\mathbb{H}^{n} \backslash B_{r}(0)\right)}=0$ and $\sup _{\mathbb{H}^{n}}|g(t)-h| \leq \varepsilon_{1}$ for all $t \geq 0$. Then

$$
\sup _{\mathbb{H}^{n}}|g(t)-h| \leq C(n, K) \cdot \mathrm{e}^{-\frac{1}{4(n+2)} t} .
$$

Moreover, $g(t) \rightarrow h$ exponentially in $C^{k}$ as $t \rightarrow \infty$ for all $k \in \mathbb{N}$.

If $g(0) \in \mathcal{M}_{\mathrm{loc}}^{\infty}\left(\mathbb{H}^{n}\right)$, solutions of (1.3) correspond to solutions of (1.2).

Theorem 1.3. Let $n \geq 4$. Let $g$ be a solution to (1.3) as in Theorem 1.1 or Theorem 1.2. Assume in addition that $g$ is smooth. Then there exists a smooth family of diffeomorphisms of $\mathbb{H}^{n}, \varphi_{0}=\mathrm{id}_{\mathbb{H}^{\mathrm{n}}}$, such that for $\tilde{g}(t):=$ $\varphi_{t}^{*} g(t)$ the family $(\tilde{g}(t))_{t \geq 0}$ is a smooth solution to (1.2) satisfying

$$
\tilde{g}(t) \rightarrow\left(\varphi_{\infty}\right)^{*} h \quad \text { in } \mathcal{M}^{\infty}\left(\mathbb{H}^{n}\right) \text { as } t \rightarrow \infty
$$

for some smooth diffeomorphism $\varphi_{\infty}$ of $\mathbb{H}^{n}$ which satisfies $\varphi_{t} \rightarrow \varphi_{\infty}$ in $C^{\infty}\left(\mathbb{H}^{n}, \mathbb{H}^{n}\right)$ as $t \rightarrow \infty$ and, if $\lim _{r \rightarrow \infty}\|g(0)-h\|_{L^{\infty}\left(\mathbb{H}^{n} \backslash B_{r}(0)\right)}=0$,

$$
\left|\varphi_{\infty}(x)-x\right| \rightarrow 0 \quad \text { as }|x| \rightarrow \infty
$$

Linearized stability of hyperbolic space under Ricci flow has been investigated before by Suneeta [11]. Ye considered stability of negatively curved manifolds on compact spaces in the paper [12]. Li and Yin [6] have shown stability of hyperbolic space in dimensions $n \geq 6$ under the assumptions that the deviation of the curvature of the initial metric from hyperbolic space decays exponentially and the initial metric is close to $h$ (in the sense of Definition 1.1). 
Similar results and methods to those found in this paper may be found in the authors' paper [8] addressing the stability of Euclidean space under Ricci flow. For further references, we refer to the introduction therein.

After this paper had appeared as a preprint, a preprint of Bamler with similar results appeared: see [1]. He does not exclude the case $n=3$.

Here we outline the proofs of the main results of this paper, and explain the structure of these proofs and of the paper.

In the first part of the paper (Sections 2 and 3) we consider the rescaled Ricci harmonic map heat flow.

There we prove short time existence using the same techniques as those presented in [8-10], see Theorem 2.1.

In Section 3, we show that the $L^{2}$-norm of $g(t)-h$ is an exponentially decaying function of time (see Theorem 3.1, Corollary 3.1). This is the key ingredient to the proofs of our stability results. The calculations to prove this depend on an eigenvalue estimate for the Laplacian on hyperbolic domains due to McKean [7] and the closeness of the evolving metric to that of hyperbolic space. In contrast to the corresponding Euclidean result [8], we need strict monotonicity of our integral quantity to establish long-time existence. The decay of the $L^{2}$-norm implies that the $C^{0}$-norm of $g(t)-h$ is exponentially decaying in time (Theorem 3.2). Interpolating between the $C^{0}$-norm and $C^{k}$-norms, $k \in \mathbb{N}$, and using interior estimates, we see that all of the $C^{k_{-}}$ norms are exponentially decaying in time (for $t \geq 1$ ). This leads to long-time existence and convergence.

In Section 4, we consider the related scaled Ricci flow $\tilde{g}(t)$ to the solution $g(t)$ obtained in Sections 2 and 3 . The two flows are related by time dependent diffeomorphisms $\varphi_{t}: \mathbb{H}^{n} \rightarrow \mathbb{H}^{n}: \tilde{g}(t):=\varphi_{t}^{*} g(t)$. As in the paper [8], we show that the estimates we obtained for $g(t)$ imply that $\tilde{g}(t) \rightarrow \psi^{*} h$ as $t \rightarrow \infty$ in the $C^{k}$-norms. Here $\psi$ is a diffeomorphism, and this diffeomorphism is the $C^{k}$-limit of the time-dependent diffeomorphisms $\varphi_{t}$ which relate the two flows. We also show (as in [8]) that $\psi \rightarrow$ id as $|x| \rightarrow \infty$, if the initial metric $g_{0}$ satisfies $g_{0}-h \rightarrow 0$ as $x \rightarrow \infty$ (see Theorem 4.2). The proofs of this section are the same (up to some minor modifications) as those of the paper [8].

In Appendix A, we gather various standard results which we use in the paper.

In Appendix B, we show that the arguments used here may be used in the Euclidean case to obtain analogous results (compare with [8]).

In Appendix C, we address conformal Ricci flow in two dimensions and obtain a result similar to the two-dimensional result in [8] without assuming that $|g-h| \rightarrow 0$ near infinity. 


\section{Existence}

We first collect some evolution equations from [9], and then treat the question of short time existence.

In the following computations we always assume that in appropriate coordinates, we have at a fixed point and at a fixed time $h_{i j}=\delta_{i j}$ and $\left(g_{i j}\right)=\operatorname{diag}\left(\lambda_{1}, \lambda_{2}, \ldots, \lambda_{n}\right), \lambda_{i}>0$.

According to [9, Lemma 2.1], we get

$$
\begin{aligned}
& \frac{\partial}{\partial t} g_{i j}= g^{a b^{h}} \nabla_{a}{ }^{h} \nabla_{b} g_{i j}-g^{k l} g_{i p} h^{p q} R_{j k q l}(h)-g^{k l} g_{j p} h^{p q} R_{i k q l}(h) \\
&+\frac{1}{2} g^{a b} g^{p q}\left({ }^{h} \nabla_{i} g_{p a}{ }^{h} \nabla_{j} g_{q b}+2^{h} \nabla_{a} g_{j p}{ }^{h} \nabla_{q} g_{i b}-2^{h} \nabla_{a} g_{j p}{ }^{h} \nabla_{b} g_{i q}\right. \\
&\left.\quad-2^{h} \nabla_{j} g_{p a}{ }^{h} \nabla_{b} g_{i q}-2^{h} \nabla_{i} g_{p a}{ }^{h} \nabla_{b} g_{j q}\right)-2(n-1) g_{i j} .
\end{aligned}
$$

Using that

$$
R_{i j k l}(h)=-\left(h_{i k} h_{j l}-h_{i l} h_{j k}\right)
$$

is the curvature tensor of hyperbolic space of sectional curvature -1 , we get

$$
\begin{aligned}
- & g^{k l} g_{i p} h^{p q} R_{j k q l}(h)-g^{k l} g_{j p} h^{p q} R_{i k q l}(h)-2(n-1) g_{i j} \\
= & g^{k l} g_{i p} h^{p q}\left(h_{j q} h_{k l}-h_{j l} h_{k q}\right) \\
& +g^{k l} g_{j p} h^{p q}\left(h_{i q} h_{k l}-h_{i l} h_{k q}\right)-2(n-1) g_{i j} \\
= & 2\left(g^{k l} g_{i j} h_{k l}-h_{i j}\right)-2(n-1) g_{i j} \\
= & 2\left(g^{k l}\left(h_{k l}-g_{k l}\right)\right) g_{i j}+2\left(g_{i j}-h_{i j}\right)
\end{aligned}
$$

and hence

Lemma 2.1. A metric $\left(g_{i j}\right)$ solving (1.3) fulfills

$$
\begin{gathered}
\frac{\partial}{\partial t} g_{i j}=g^{a b^{h}} \nabla_{a}{ }^{h} \nabla_{b} g_{i j} \\
+2 g_{i j}\left(g^{k l}\left(h_{k l}-g_{k l}\right)\right)+2\left(g_{i j}-h_{i j}\right) \\
+\frac{1}{2} g^{a b} g^{p q}\left({ }^{h} \nabla_{i} g_{p a}{ }^{h} \nabla_{j} g_{q b}+2^{h} \nabla_{a} g_{j p} \nabla_{q} g_{i b}-2^{h} \nabla_{a} g_{j p}{ }^{h} \nabla_{b} g_{i q}\right. \\
\left.-2^{h} \nabla_{j} g_{p a}{ }^{h} \nabla_{b} g_{i q}-2^{h} \nabla_{i} g_{p a}{ }^{h} \nabla_{b} g_{j q}\right)
\end{gathered}
$$


For later use, we derive the evolution equation of $Z:=|g-h|^{2}$. We use the following notation in this Lemma and in the rest of the paper.

Notation: We write $O_{n}(\varepsilon)$ for any number in $\mathbb{R}$ satisfying $\left|O_{n}(\varepsilon)\right| \leq c(n) \varepsilon$ where $c(n)$ is some fixed big number $(n \in \mathbb{N})$. Similarly $\left|O_{n, m}(\varepsilon)\right| \leq c(n, m) \varepsilon$ where $c(n, m)$ is some fixed big number $(n, m \in \mathbb{N})$.

Lemma 2.2. Let $g \in \mathcal{M}^{\infty}\left(\mathbb{H}^{n},(0, T)\right), T>0$, be a solution to (1.3) which is $\varepsilon$-close to the hyperbolic metric $h$ of sectional curvature -1 . Assume that $\varepsilon>0$ is sufficiently small, $\varepsilon \leq \varepsilon_{0}(n)$. Then

$$
\begin{aligned}
\frac{\partial}{\partial t}|g-h|^{2} \leq & g^{i j} \nabla_{i} \nabla_{j}|g-h|^{2}-\left(2-O_{n}(\varepsilon)\right)|\nabla(g-h)|^{2} \\
& +\left(4+O_{n}(\varepsilon)\right)|g-h|^{2}
\end{aligned}
$$

where we write $\nabla$ for ${ }^{h} \nabla$.

Proof. Note that the norm of a tensor $Z$ of order $m$ fulfills

$$
\left.{ }^{h}|Z|^{2} \equiv|Z|^{2} \leq\left(1+O_{n, m}(\varepsilon)\right) \cdot{ }^{g}|Z|^{2} \leq\left(1+O_{n, m}(\varepsilon)\right)\right) \cdot{ }^{h}|Z|^{2}
$$

for $g$ which are $\varepsilon$-close to $h$. Choose coordinates such that $h_{i j}=\delta_{i j}$ and $g_{i j}=\lambda_{i} \delta_{i j}$. We use $*$ similarly as in $[4$, Ch. 13] to denote contractions with respect to $h, g$ or their inverses. Let $Z=g-h$. Lemma 2.1 yields

$$
\begin{aligned}
\frac{\partial}{\partial t}|Z|^{2} \equiv & \frac{\partial}{\partial t}|g-h|^{2}=2 \sum_{i}\left(g_{i i}-h_{i i}\right)\left(\frac{\partial}{\partial t} g_{i i}\right) \\
= & g^{i j} \nabla_{i} \nabla_{j}|g-h|^{2}-\left(2-O_{n}(\varepsilon)\right)|\nabla g|^{2} \\
& +2 \sum_{i}\left(g_{i i}-h_{i i}\right)\left[2\left(g_{i i}-h_{i i}\right)-2 g_{i i} \sum_{k}\left(g^{k k}\left(g_{k k}-h_{k k}\right)\right)\right] \\
& +\sum_{i}\left(g_{i i}-h_{i i}\right)(\nabla g * \nabla g)_{i i}
\end{aligned}
$$




$$
\begin{aligned}
\leq & g^{i j} \nabla_{i} \nabla_{j}|g-h|^{2}-\left(2-O_{n}(\varepsilon)\right)|\nabla(g-h)|^{2} \\
& +4 \sum_{i}\left(g_{i i}-h_{i i}\right)\left[\left(g_{i i}-h_{i i}\right)-g_{i i} \sum_{k}\left(g^{k k}\left(g_{k k}-h_{k k}\right)\right)\right] .
\end{aligned}
$$

Let us examine the zeroth-order term $S$ of the above equation.

$$
\begin{aligned}
S & =4 \sum_{i}\left(g_{i i}-h_{i i}\right)\left[\left(g_{i i}-h_{i i}\right)-g_{i i} \sum\left(g^{k k}\left(g_{k k}-h_{k k}\right)\right)\right] \\
& =4 \sum_{i}\left(\lambda_{i}-1\right)^{2}-4 \sum_{i} \lambda_{i}\left(\lambda_{i}-1\right) \sum_{k}\left(1-\frac{1}{\lambda_{k}}\right) \\
& \leq\left(4+O_{n}(\varepsilon)\right)|Z|^{2}-4\left(\sum_{i}\left(\lambda_{i}-1\right)\right)^{2} .
\end{aligned}
$$

The claim follows.

We use this to show that we can solve Dirichlet problems for (1.3) on a short-time interval. In the following we pick a point $p_{0}$ and fix it throughout. For simplicity of notation we will denote this point with 0 . All balls $B_{R}(0)$ are geodesic balls with respect to the hyperbolic metric $h$.

The following result also extends readily to (1.3) on all of $\mathbb{H}^{n}$ provided that a non-compact maximum principle is applicable.

Corollary 2.1. Let $g \in \mathcal{M}_{0}^{\infty}\left(B_{R}(0),[0, T)\right), 0<T<\infty$, be a solution to (1.3) on $B_{R}(0) \subset \mathbb{H}^{n}$ instead of $\mathbb{H}^{n}$ with $\left.g(t)\right|_{\partial B_{R}(0)}=\left.h\right|_{\partial B_{R}(0)}$. Let $0<$ $\delta$. Then there exists $\varepsilon_{1}=\varepsilon_{1}(n, T, \delta)>0$ such that $\sup _{B_{R}(0)}|g(0)-h| \leq \varepsilon_{1}$ implies

$$
\sup _{B_{R}(0) \times[0, T)}|g-h| \leq \delta .
$$

Proof. Fix $\varepsilon_{1}:=\delta \mathrm{e}^{-5 T}$, and let $\varepsilon$ be given with $\varepsilon \leq \varepsilon_{1}$. Without loss of generality $\sqrt{\delta} \leq \varepsilon_{0}$ of Lemma 2.2, and $O_{n}(\varepsilon)$ appearing on the right-hand side of $(2.1)$ satisfies $O_{n}(\varepsilon) \leq 1$. Then Lemma 2.2 and the maximum principle imply that

$$
\sup _{B_{R}(0)}|g(t)-h|^{2} \mathrm{e}^{5(T-t)} \leq \sup _{B_{R}(0)}\left|g_{0}-h\right|^{2} \mathrm{e}^{5 T} \leq \varepsilon_{1} \mathrm{e}^{5 T}=\delta
$$

as long as $\sup _{B_{R}(0)}|g(t)-h| \leq \varepsilon_{0}$ remains true. But $\sup _{B_{R}(0)}|g(t)-h|^{2} \leq$ $\delta$ implies $\sup _{B_{R}(0)}|g(t)-h| \leq \sqrt{\delta} \leq \varepsilon_{0}$ and hence the condition $\sup _{B_{R}(0)}$ $|g(t)-h| \leq \varepsilon_{0}$ is not violated for all $t \in[0, T]$. The result follows. 
If solutions to (1.3) stay sufficiently close to the hyperbolic metric $h$ of sectional curvature -1 , they exist for all times.

Theorem 2.1. For all $n \in \mathbb{N}$ there exists a $\tilde{\delta}(n)>0$ such that the following holds. Let $0<\varepsilon<\delta \leq \tilde{\delta}(n)$. Then every metric $g_{0} \in \mathcal{M}^{0}\left(\mathbb{H}^{n}\right)$ with $\| g_{0}-$ $h \|_{L^{\infty}} \leq \varepsilon$ has a $\delta$-maximal solution $g \in \mathbb{M}_{0}^{\infty}\left(\mathbb{H}^{n},\left[0, T_{g_{0}}\right)\right)$ to $(1.3)$, where $T_{g_{0}}>0$ and $\|g(t)-h\|_{L^{\infty}}<\delta$ for all $t \in\left[0, T_{g_{0}}\right)$. The solution is $\delta$-maximal in the following sense. Either $T_{g_{0}}=\infty$ and $\|g(t)-h\|_{L^{\infty}}<\delta$ for all $0 \leq t$ or we can extend $g$ to a solution on $\mathbb{H}^{n} \times\left[0, T_{g_{0}}+\tau\right)$, for some $\tau=\tau(n)>0$, and $\left\|g\left(T_{g_{0}}\right)-h\right\|_{L^{\infty}}=\delta$.

Proof. The proof follows directly from the corresponding proofs in [8-10]: we mollify $g_{0}$ and obtain $g_{0}^{i}, i \in \mathbb{N}$. Then we consider $g_{0}^{i, R}:=\eta g_{0}^{i}+(1-\eta) h, R \geq$ 1 , where $\eta: \mathbb{H}^{n} \rightarrow \mathbb{H}^{n}$ is a smooth function fulfilling $\eta=1$ in $B_{R}(0), \eta=0$ outside $B_{2 R}(0)$ and $|\nabla \eta| \leq 2 / R$. Hence $\left\|g_{0}^{i, R}-h\right\|_{L^{\infty}} \leq\left\|g_{0}^{i}-h\right\|_{L^{\infty}} \leq \| g_{0}-$ $h \|_{L^{\infty}\left(\mathbb{H}^{n}\right)} \leq \varepsilon$. Arguing as in [8-10] (see Section 3 of [9]), and using that $g_{0}^{i, R}$ is $\tilde{\delta}(n)$ close to $h$, we see that there exist solutions $g^{i, R} \in \mathcal{M}^{\infty}\left(B_{3 R}(0),[0, \tau]\right)$ with $0<\tau=\tau(n)<\infty$ to $(1.3)$ on $B_{3 R}(0)$ with $g^{i, R}(0)=g_{0}^{i, R}$ on $B_{3 R}(0)$ and $g^{i, R}=h$ on $\partial B_{3 R}(0) \times[0, \tau]$. From Lemma 5.1 of $[10]$ we see that we have interior estimates of the form $\left|\nabla^{j} g^{i, R}\right|^{2} \leq c / t^{j}$ on balls of radius $R / 2$ for all $t \in[0, \tau]$ for all $j \in \mathbb{N}$. Taking a diagonal subsequence and using the theorem of Arzelà-Ascoli, we obtain a solution $g \in \mathcal{M}^{\infty}\left(\mathbb{H}^{n},(0, \tau]\right)$. Furthermore, $g(t) \rightarrow g_{0}$ as $t \rightarrow 0$ in the $C_{\mathrm{loc}}^{0}$-norm as we see from Theorem 5.2 in [10].

If $\|g(t)-h\|_{L^{\infty}}<\delta$ for all $t \in[0, \tau]$, then we may repeat this argument in view of the fact that $\delta \leq \tilde{\delta}(n)$. By induction we obtain a solution $g \in$ $M_{0}^{\infty}\left(\mathbb{H}^{n},[0, S)\right)$ where either

(a) $S=\infty$ and $\|g(t)-h\|_{L^{\infty}}<\delta$ for all $t>0$, or

(b) $S=N \tau$ for some $N \in \mathbb{N}$ and $\|g(t)-h\|_{L^{\infty}}<\delta$ for all $t \in[0,(N-1) \tau]$ but there exists at least one time $\tilde{t} \in((N-1) \tau, N \tau]$ with $\| g(\tilde{t})-$ $h \|_{L^{\infty}}=\delta$.

In the case (a), we set $T_{g_{0}}=\infty$ and we are finished.

So assume we are in case (b) for the rest of the argument. In view of Lemma 2.2, the maximum principle, and the way we constructed our solutions, we see that in fact there is a first time $T_{g_{0}} \in((N-1) \tau, N \tau]$ with $\left\|g\left(T_{g_{0}}\right)-h\right\|_{L^{\infty}}=\delta$ and $\|g(t)-h\|_{L^{\infty}}<\delta$ for all $t \in\left[0, T_{g_{0}}\right)$. Using that $g\left(T_{g_{0}}\right)$ is $\delta$ close to $h$ and $\delta \leq \tilde{\delta}$ we may repeat the first part of the proof to obtain a solution defined on $\left[0, T_{g_{0}}+\tau\right]$. This completes the proof. 
Proposition 2.1. Assume everything is as in Theorem 2.1, and $\hat{T}>0$ be given. If we choose $\varepsilon=\varepsilon(n, \delta, \hat{T})>0$ small enough in the above Theorem, then the solution $g \in \mathbb{M}_{0}^{\infty}\left(\mathbb{H}^{n},\left[0, T_{g_{0}}+\tau\right)\right)$ from Theorem 2.1 satisfies $T_{g_{0}} \geq \hat{T}$.

Proof. By Corollary 2.1, we can choose $\varepsilon=\varepsilon(n, \delta, \hat{T})$ small enough so that $\|g(t)-h\|_{L^{\infty}}<\delta$ for all $t \in[0, \hat{T}] \cap\left[0, T_{g_{0}}\right]$. Theorem 2.1 yields a contradiction if $T_{g_{0}}<\hat{T}$.

\section{Convergence}

Convergence is based on a Lyapunov function.

Theorem 3.1. Let $n \geq 4$. There exists $\delta_{0}=\delta_{0}(n)>0$ such that the following holds. Let $g \in \mathcal{M}^{\infty}\left(B_{R},[0, T)\right)$ be a solution to (1.3) with $g=h$ on $\partial B_{R}(0) \times[0, T)$ and assume that $\sup _{B_{R}(0) \times[0, T)}|g-h| \leq \delta_{0}$. Then we have

$$
\int_{B_{R}(0)}|g(t)-h|^{2} d \operatorname{vol}_{h} \leq \mathrm{e}^{-\alpha t} \int_{B_{R}(0)}|g(0)-h|^{2} d \operatorname{vol}_{h}
$$

for $\alpha(n):=\left(2(n-1)^{2}-17\right) / 4 \geq \frac{1}{4}$.

Proof. Assume that $\delta_{0}$ is such that $g$ is $\varepsilon(n)$-close to $h$ for some sufficiently small $1 \geq \varepsilon(n)>0$. We compute for $Z=g-h$, using Lemma 2.2,

$$
\begin{aligned}
\frac{\partial}{\partial t} \int_{B_{R}(0)}|Z|^{2} d \mathrm{vol}_{h} \leq & \int_{B_{R}(0)} g^{i j} \nabla_{i} \nabla_{j}|Z|^{2}-\left(2-O_{n}(\varepsilon)\right)|\nabla Z|^{2} \\
& +\left(4+O_{n}(\varepsilon)\right)|Z|^{2} d \operatorname{vol}_{h} \\
= & \int_{\partial B_{R}(0)} \nu_{i} g^{i j} \nabla_{j}|Z|^{2}-\int_{B_{R}(0)} \nabla_{j} g^{j k} \nabla_{k}|Z|^{2} d \operatorname{vol}_{h} \\
& +\int_{B_{R}(0)}-\left(2-O_{n}(\varepsilon)\right)|\nabla Z|^{2}+\left(4+O_{n}(\varepsilon)\right)|Z|^{2} d \mathrm{vol}_{h} \\
\leq & \int_{B_{r}(0)}-\left.\left(2-O_{n}(\varepsilon)\right)|\nabla| Z\right|^{2}+\left(4+O_{n}(\varepsilon)\right)|Z|^{2} d \operatorname{vol}_{h}
\end{aligned}
$$

where we used that $\left.\left.\left|\nabla_{i} g^{i j} \nabla_{j}\right| Z\right|^{2}\left|\leq O_{n}(\varepsilon)\right| \nabla Z\right|^{2}$ and that on $\partial B_{R}(0)$ the gradient $\nabla|Z|^{2}$ is anti-parallel to the outer unit normal $\nu$. Furthermore we apply Kato's inequality $|\nabla| Z||^{2} \leq|\nabla Z|^{2}$ which is valid whenever $|Z| \neq 0$ and 
for Sobolev functions. More explicitly

$$
\begin{aligned}
\left.\left|\nabla_{i} g^{i j} \nabla_{j}\right| Z\right|^{2} \mid & \leq c(n)|\nabla g||Z||\nabla Z| \\
& \leq c(n)|Z||\nabla Z|^{2} \\
& \leq O_{n}(\varepsilon)|\nabla Z|^{2}
\end{aligned}
$$

since $|Z|=|g-h| \leq O_{n}(\varepsilon)$ and $|\nabla g|=|\nabla(g-h)|=|\nabla Z|$.

Using McKean's inequality [7] for the first eigenvalue

$$
\sigma_{1} \geq \frac{(n-1)^{2}}{4}
$$

on hyperbolic domains we see

$$
\begin{aligned}
\frac{\partial}{\partial t} \int_{B_{R}(0)}|Z|^{2} d \operatorname{vol}_{h} & \leq \int_{B_{R}(0)}-\left.\left(2-O_{n}(\varepsilon)\right)|\nabla| Z\right|^{2}+\left(4+O_{n}(\varepsilon)\right)|Z|^{2} d \operatorname{vol}_{h} \\
& \leq \frac{\left(8-(n-1)^{2}+c(n) \varepsilon\right)}{2} \int_{B_{R}(0)}|Z|^{2} d \operatorname{vol}_{h} .
\end{aligned}
$$

Assuming that $\varepsilon<1 /(2 c(n))$, we can choose

$$
\alpha:=\left(2(n-1)^{2}-17\right) / 4 .
$$

Since for the proof of existence of a solution to (1.3) we use Dirichlet problems as above, this monotonicity extends to the constructed solutions on $\mathbb{H}^{n} \times[0, T)$ : let $g^{i, R}(t)$ be as in Theorem 2.1. Then we get

$$
\left\|g^{i, R}(t)-h\right\|_{L^{2}\left(B_{3 R}(0)\right)}^{2} \leq \mathrm{e}^{-\alpha t}\left\|g_{0}^{i, R}-h\right\|_{L^{2}\left(B_{3 R}(0)\right)}^{2} \leq \mathrm{e}^{-\alpha t}\left\|g_{0}-h\right\|_{L^{2}\left(\mathbb{H}^{n}\right)}^{2}
$$

As $R \rightarrow \infty$, we obtain

Corollary 3.1. Let $n \geq 4$ and $T>0$ be given. Assume that $g_{0} \in \mathcal{M}^{\infty}\left(\mathbb{H}^{n}\right)$ satisfies $\left\|g_{0}-h\right\|_{L^{2}\left(\mathbb{H}^{n}\right)}<\infty$. Then there exists $\varepsilon_{0}=\varepsilon_{0}(n, T)$ such that, if $\sup _{\mathbb{H}^{n}}\left|g_{0}-h\right| \leq \varepsilon_{0}$ then a solution $g \in \mathcal{M}^{\infty}\left(\mathbb{H}^{n},[0, T)\right)$ to $(1.3)$ with $g(\cdot, 0)=$ $g_{0}(\cdot)$ exists and $\sup _{\mathbb{H}^{n} \times[0, T)}|g-h| \leq \delta_{0}$, where $\delta_{0}$ is as in Theorem 3.1. Furthermore we have the estimate

$$
\|g(t)-h\|_{L^{2}\left(\mathbb{H}^{n}\right)}^{2} \leq \mathrm{e}^{-\alpha t}\left\|g_{0}-h\right\|_{L^{2}\left(\mathbb{H}^{n}\right)}^{2}
$$

for all $t \in[0, T)$, where $\alpha=\alpha(n) \geq \frac{1}{4}$. 
Proof. Existence and closeness to $h$ follow from Corollary 2.1, Proposition 2.1 and Theorem 2.1.

Using the gradient estimate we see that the exponential convergence of the $L^{2}$-norm of $|g-h|$ also implies exponential convergence in the sup-norm, compare [8, Lemma 7.1].

Theorem 3.2. Let $n \geq 4$. Assume that $g \in \mathcal{M}^{\infty}\left(\mathbb{H}^{n},[0, T)\right)$ is a solution to (1.3) with $\|g(0)-h\|_{L^{2}\left(\mathbb{H}^{n}\right)}=: K<\infty$, $\sup _{\mathbb{H}^{n} \times[0, T)}|g-h| \leq \delta_{0}$ and

$$
\|g(t)-h\|_{L^{2}\left(\mathbb{H}^{n}\right)}^{2} \leq \mathrm{e}^{-\alpha t}\|g(0)-h\|_{L^{2}\left(\mathbb{H}^{n}\right)}^{2},
$$

where $\delta_{0}$ is as in Theorem 3.1. Then

$$
\sup _{\mathbb{H}^{n}}|g(t)-h| \leq C(n, K) \mathrm{e}^{-\beta t},
$$

where $\beta=\frac{\alpha}{n+2}=\frac{2(n-1)^{2}-17}{4(n+2)}>0$.

Proof. We can assume w.l.o.g. that $\delta_{0}<1$. We choose $\tau:=\frac{n+1}{\alpha} \ln \left(\delta_{0}^{-1}\right)>0$. Note that this implies

$$
\sup _{\mathbb{H}^{n}}|g(t)-h| \leq \mathrm{e}^{-\beta t}
$$

for $t \in[0, \tau)$ and $\beta:=\alpha /(n+1)$. By the interior estimates of the form $\left|{ }^{h} \nabla g(t)\right| \leq c \cdot t^{-1 / 2}$, there exists a constant $C^{\prime}=C^{\prime}(n)$, such that

$$
\left|{ }^{h} \nabla g(\cdot, t)\right|^{h} \leq C^{\prime}
$$

for $t \in[\tau, T)$. Fix such a $t \in[\tau, T)$. Let $\gamma:=\sup _{\mathbb{H}^{n}}|g(t)-h|$ and choose a point $p_{0} \in \mathbb{H}^{n}$ such that $\left|g\left(p_{0}, t\right)-h\left(p_{0}\right)\right| \geq \frac{1}{2} \gamma$. By the gradient estimate, we have

$$
|g(\cdot, t)-h| \geq \frac{1}{4} \gamma
$$

on $B_{\gamma /\left(4 C^{\prime}\right)}\left(p_{0}\right)$. This implies

$$
\|g(t)-h\|_{L^{2}\left(\mathbb{H}^{n}\right)}^{2} \geq \omega_{n}\left(C^{\prime}\right)^{-n}\left(\frac{\gamma}{4}\right)^{n+2},
$$

where $\omega_{n}$ is the measure of the unit ball in $\mathbb{R}^{n}$. This yields

$$
\gamma \leq 4\left(C^{\prime}\right)^{\frac{n}{n+2}}\left(\frac{K^{2}}{\omega_{n}}\right)^{\frac{1}{n+2}} \mathrm{e}^{-\frac{\alpha}{n+2} t} .
$$

Choosing $C(n, K)=1+4\left(C^{\prime}\right)^{\frac{n}{n+2}}\left(\frac{K^{2}}{\omega_{n}}\right)^{\frac{1}{n+2}}$ we have $(3.1)$. 
This sup-estimate allows us to construct a solution which exists for all times.

Theorem 3.3. Let $n \geq 4$. For all $K>0$ there exists $\varepsilon_{1}=\varepsilon_{1}(n, K)>0$ such that the following holds. Let $g_{0} \in \mathcal{M}^{\infty}\left(\mathbb{H}^{n}\right)$ satisfy $\left\|g_{0}-h\right\|_{L^{2}\left(\mathbb{H}^{n}\right)} \leq K$ and $\sup _{\mathbb{H}^{n}}\left|g_{0}-h\right| \leq \varepsilon_{1}$. Then there exists a solution $g \in \mathcal{M}^{\infty}\left(\mathbb{H}^{n},[0, \infty)\right)$ to (1.3) with $g(0)=g_{0}$ such that

$$
\sup _{\mathbb{H}^{n}}|g(t)-h| \leq C(n, K) \mathrm{e}^{-\beta t}
$$

for $\beta=\beta(n)$ as in Theorem 3.2.

Proof. According to Theorem 2.1, we obtain existence for all times if we can prove the estimate $\|g(t)-h\|_{L^{\infty}} \leq \tilde{\delta}=\tilde{\delta}_{\text {Thm. } 2.1}$ for all $t$ for any a priori solution (that is, we must prove the estimate for all $t$ that the solution is defined). Given any $T>0$, we can choose $\varepsilon(n, T)>0$ small enough so that such an estimate will hold, in view of Proposition 2.1 and Theorem 2.1 for $0 \leq t<T$. Theorem 3.1 implies integral bounds which combined with Theorem 3.2 yields $|g(t)-h| \leq \tilde{\delta}$ if $t \geq T$ and $T$ is chosen sufficiently large. Choose $T$ and $\varepsilon(n, T)$ so that both of these requirements are satisfied.

This implies long-time existence.

Theorem 3.2 also implies (3.2) for $t \geq T$. Fixing $C(n, K)$ such that $C(n, K) \leq \delta \cdot \mathrm{e}^{\beta T}$ we obtain (3.2) for all times.

By interpolation the exponential decay extends to higher derivatives of the evolving metric.

Theorem 3.4. Let $n \geq 4$. Let $g_{0} \in \mathcal{M}^{\infty}\left(\mathbb{H}^{n}\right)$ and $g \in \mathcal{M}^{\infty}\left(\mathbb{H}^{n},[0, \infty)\right)$ be as in Theorem 3.3. We have additionally

$$
\sup _{\mathbb{H}^{n}}\left|{ }^{h} \nabla^{j} g(t)\right| \leq C\left(n, j, K,\left(\beta_{j}\right)\right) \mathrm{e}^{-\beta_{j} t}
$$

where $0<\beta_{j}<\beta(n), \beta(n)$ as in Theorem 3.2, is arbitrary. In particular,

$$
\lim _{t \rightarrow \infty} \sup _{\mathbb{H}^{n}}\|g(t)-h\|_{C^{k}\left(\mathbb{H}^{n}\right)}=0
$$

where $\|S\|_{C^{k}}:=\sum_{i=0}^{n} \sup _{\mathbb{H}^{n}}\left|\nabla^{j} S\right|^{2}$.

Proof. From the interior estimates in Lemma A.1, we have $\sup _{\mathbb{H}^{n}}\left|\nabla^{j} g\right|^{2}(t) \leq$ $c(n, j) /(t-L)$, for all $t \in[L, L+1]$. In particular, $\sup _{\mathbb{H}^{n}}\left|\nabla^{j} g\right|^{2}(L+1) \leq$ 
$c(n, j)$. Hence, as $L>0$ was arbitrary, we get

$$
\sup _{\mathbb{H}^{n}}\left|\nabla^{j} g\right|^{2}(\cdot, t) \leq c(n, j)
$$

for all $t \geq 1$. Interpolating on a ball of radius one (see Lemma A.6) gives us

$$
\sup _{\mathbb{H}^{n}}\left|\nabla^{j} g\right|^{2}(t) \leq \tilde{c}(n, j)\left(\sup _{\mathbb{H}^{n}}|g(t)-h|\right)^{\frac{1}{2^{j-1}}} \leq C(n, j, K) \mathrm{e}^{-\frac{\beta}{2^{j-1}} t}
$$

in view of (3.3) and (3.1). Iterated interpolation yields the result, see e. g., [8].

Proof of Theorem 1.1. As the decay of $|g(t)-h|$ as $t \rightarrow \infty$ obtained in this section does not depend on the smoothness of $g_{0}$, we can approximate $g_{0} \in$ $\mathcal{M}^{0}\left(\mathbb{H}^{n}\right)$ and pass to a limit to obtain Theorem 1.1.

Proof of Theorem 1.2. Local closeness estimates (see Lemma A.2) show that

$$
\lim _{r \rightarrow \infty}\|g(t)-h\|_{L^{\infty}\left(\mathbb{H}^{n} \backslash B_{r}(0)\right)}=0
$$

is preserved during the flow, even uniformly on compact time intervals. Hence

$$
\max \left\{|g(t)-h|^{2}-\delta, 0\right\} \equiv\left(|g-h|^{2}-\delta\right)_{+}
$$

has compact support on $\mathbb{H}^{n} \times[0, K]$ for all $K<\infty$ and we may consider the integral $I_{\delta}:=\left\|\left(|g-h|^{2}-\delta\right)_{+}\right\|_{L^{1}\left(\mathbb{H}^{n}\right)}$ for any $\delta>0$, which is similar to the integral $I_{\delta}^{m, p}$ defined in [8, Theorem 6.1] or to $I_{\delta}^{p}$ in Appendix B. The techniques of the proof of Theorem 3.1 and approximations as in [8, Th. 6.1] imply for $R \gg 1$ that

$$
\begin{aligned}
I_{\delta}^{R}(t) & :=\left\|\left(|g(t)-h|^{2}-\delta\right)_{+}\right\|_{L^{1}\left(B_{R}(0)\right)} \leq \mathrm{e}^{-\alpha t} \cdot I_{\delta}^{R}(0) \\
& \leq \mathrm{e}^{-\alpha t} \cdot\|g(0)-h\|_{L^{2}\left(\mathbb{H}^{n}\right)}^{2} .
\end{aligned}
$$

The rest of the proof is similar to the proof of Theorem 1.1.

\section{Getting back to Ricci Flow}

Theorem 4.1. Assume that $g \in \mathcal{M}^{\infty}\left(\mathbb{H}^{n},[0, \infty)\right)$ is a solution to (1.3) coming from Theorem 3.3. Then there exists a smooth map $\varphi: \mathbb{H}^{n} \times[0, \infty)$ 
$\rightarrow \mathbb{H}^{n}$ such that $\varphi(\cdot, t)=: \varphi_{t}: \mathbb{H}^{n} \rightarrow \mathbb{H}^{n}$ is a diffeomorphism, $\varphi_{0}=\mathrm{id}$ and $\tilde{g}(\cdot, t):=\left(\varphi_{t}\right)^{*} g(\cdot, t)$ is a smooth solution to the scaled Ricci flow

$$
\frac{\partial}{\partial t} g=-2 \operatorname{Ric}-2(n-1) g
$$

with $\tilde{g}_{t} \rightarrow g_{0}$ as $t \searrow 0$. Furthermore there exists a smooth diffeomorphism $\psi:$ $\mathbb{H}^{n} \rightarrow \mathbb{H}^{n}$ with $\varphi_{t} \rightarrow \psi$ as $t \rightarrow \infty$ and $\tilde{g}_{t} \rightarrow \psi^{*} g_{0}$ as $t \rightarrow \infty$. Here convergence is in $C^{k}$ on $\mathbb{H}^{n}$ for all $k$.

Proof. This argument is the same as in Lemma 9.1 and Theorem 9.2 of [8] with some minor differences. We explain here where the argument of [8] must be modified in order for it to work in this case.

As explained in Lemma 9.1 in [8], we can construct smooth maps $\varphi$ : $\mathbb{H}^{n} \times[0, \infty) \rightarrow \mathbb{H}^{n}$ such that

$$
\begin{cases}\frac{\partial}{\partial t} \varphi^{\alpha}(x, t)=V^{\alpha}(\varphi(x, t), t), & (x, t) \in \mathbb{H}^{n} \times[0, \infty), \\ \varphi(x, 0)=x, & x \in \mathbb{H}^{n},\end{cases}
$$

where $V^{\alpha}(y, t):=-g^{\beta \gamma}\left({ }^{g} \Gamma_{\beta \gamma}^{\alpha}-{ }^{h} \Gamma_{\beta \gamma}^{\alpha}\right)(y, t)$ and $\varphi_{t}:=\varphi(\cdot, t): \mathbb{H}^{n} \rightarrow \mathbb{H}^{n}$ are diffeomorphisms. (Compared to [8], we have changed the sign in the definition of $V$ in order to correct a typo there.) This part of the proof is the same. A direct calculation shows that $\tilde{g}(t):=\varphi_{t}^{*} g(t)$ solves the scaled Ricci flow equation.

In Theorem 9.2 of [8] it is shown that $\varphi_{t} \rightarrow \varphi_{\infty}$ as $t \rightarrow \infty$ where $\varphi_{\infty}$ : $\mathbb{H}^{n} \rightarrow \mathbb{H}^{n}$ is a smooth diffeomorphism, and the convergence is in $C^{k}$ (for all $k)$ on $\mathbb{H}^{n}$. The proof of this is carried out in three steps.

In step 1 it is shown that $\left|\frac{\partial}{\partial t} \varphi_{t}(x)\right| \leq \frac{1}{t^{r}}$ for some $r>1$, for all $t \geq 1$, and $\left|\varphi_{t}(x)-x\right| \leq c$ for all $t$.

In step 2, the existence of a smooth function $\varphi_{\infty}: \mathbb{H}^{n} \rightarrow \mathbb{H}^{n}$ with $\varphi_{t} \rightarrow$ $\varphi_{\infty} \equiv \psi$ as $t \rightarrow \infty$ is shown.

The proofs of steps one and two carry over to this situation without any changes.

In step 3 , it is shown that $\varphi_{\infty}$ is a diffeomorphism. This proof carries over with some minor modifications which we describe in the rest of the proof here. 
Letting $l(t):=\varphi_{t}^{*} g(t)$, we know that $l$ solves the scaled Ricci flow (1.2) on $\mathbb{H}^{n}$, and that

$$
\begin{aligned}
& \sup _{\mathbb{H}^{n}} l(t)|\operatorname{Ric}(l(t))+2(n-1) l(t)| \\
& \quad=\sup _{\mathbb{H}^{n}} g(t)|\operatorname{Ric}(g(t))+2(n-1) g(t)| \\
& \leq \sup _{\mathbb{H}^{n}} g(t)|\operatorname{Ric}(g(t))-\operatorname{Ric}(h)|+\sup _{\mathbb{H}^{n}} g(t)|\operatorname{Ric}(h)+2(n-1) g(t)| \\
& \quad=\sup _{\mathbb{H}^{n}} g(t)|\operatorname{Ric}(g(t))-\operatorname{Ric}(h)|+\sup _{\mathbb{H}^{n}} g(t)|-2(n-1) h+2(n-1) g(t)| \\
& \leq \mathrm{e}^{-\beta t}
\end{aligned}
$$

for all $t>0$ form some $\beta>0$. The last inequality may be seen easily by writing the terms in local coordinates and then using the estimates of Theorem 3.4.

Hence $l(t)$ converges locally uniformly (smoothly) to a smooth metric $l_{\infty}$ on $\mathbb{H}^{n}$ as explained in [8].

Choose geodesic coordinates for $h$ centred at $y$ in $B_{1}(y)$. Now using the definition of $l$, and the uniform convergence of $l$ we get

$$
\begin{aligned}
\frac{1}{c} \delta_{\alpha \beta} \leq l_{\alpha \beta}(x, t) & =\frac{\partial \varphi_{t}^{s}}{\partial y^{\alpha}}(x, t) \frac{\partial \varphi_{t}^{k}}{\partial y^{\beta}}(x, t) g_{s k}\left(\varphi_{t}(x), t\right) \\
& \leq(1+\tilde{\varepsilon}) \frac{\partial \varphi_{t}^{s}}{\partial y^{\alpha}} \frac{\partial \varphi_{t}^{k}}{\partial y^{\beta}}(x, t) h_{s k}\left(\varphi_{t}(x)\right) \\
& \leq c(1+\tilde{\varepsilon})\left(D \varphi_{t}\right)\left(D \varphi_{t}\right)^{T}(x, t) .
\end{aligned}
$$

In particular, we see that $\operatorname{det}\left(D \varphi_{t}\right)^{2}(x) \geq \frac{1}{(1+\tilde{\varepsilon})^{n} c}>0$ for $x \in B_{1}(y)$, where $D f$ is the Jacobian of $f$. As explained in [8], this shows that $\varphi_{\infty}$ is a diffeomorphism.

Theorem 4.2. Let everything be as in the above Theorem 4.1, with the extra assumption that $\sup _{\left(\mathbb{H}^{n} \backslash B_{r}(0)\right)}\left|g_{0}-h\right| \rightarrow 0$ as $r \rightarrow \infty$. Then the diffeomorphism $\psi$ appearing in the above Theorem satisfies $\psi \rightarrow \mathrm{id}$ as $x \rightarrow \infty$ (in $C^{k}$ for all $k$ ). In particular, for every $\eta>0$, there exists an $R>0$ such that

$$
\sup _{\mathbb{H}^{n} \backslash B_{R}(0)}\left|\varphi_{t}(x)-x\right| \leq \eta,
$$

for all $t$.

Proof. The proof is completely analogous to the proof of Lemma 9.3 in [8]. Let $\eta>0$. From Lemma A.2 and the estimates of Theorem 3.3 we can choose 
$R>0$ large so that

$$
|g(t)-h| \leq \eta \text { on } \mathbb{H}^{n} \backslash B_{R}(0),
$$

for all $t>0$. From the interior estimates of [10] (see Lemma A.1) we get

$$
\left|\nabla^{2} g\right| \leq \frac{c}{t}
$$

for $t \in[0,1]$, and hence

$$
\left|\nabla^{2} g\right| \leq \frac{c}{t}
$$

for all $t \in[0, \infty)$, in view of Theorem 3.3 and interpolation with higher-order derivatives, see Lemmas A.1 and A.6. Interpolating between the $C^{0}$-norm and the $C^{2}$-norm on a ball of radius one for $t \leq 1$ (see Lemma A.6) we get

$$
|\nabla g| \leq \frac{\sqrt{c} \sqrt{\eta}}{\sqrt{t}}
$$

for all $t \in[0, \infty)$ on $\mathbb{H}^{n} \backslash B_{R}(0)$. Arguing as in Step 1 of the above theorem gives us the result.

\section{A. Scaling and interior estimates}

Lemma A.1. Let $\left(\mathbb{H}^{n}, g(t)\right)_{t \in[0, T)}$ be a solution to equation (1.3), with $|g(t)-h| \leq \varepsilon(n)$ for $\varepsilon(n)>0$ small enough. Then

$$
\sup _{\mathbb{H}^{n}}\left|{ }^{h} \nabla^{j} g(\cdot, t)\right|^{2} \leq \frac{c(j, n)}{t^{j}}
$$

for all $t \leq \min \{1, T\}$.

Proof. This is Theorem 4.3 in [10].

Lemma A.2. Fix a point $p_{0} \in \mathbb{H}^{n}$ and let $g \in \mathcal{M}^{\infty}\left(\mathbb{H}^{n},[0, T)\right)$, where $T \in$ $(0, \infty]$, be a solution to $(1.3)$ such that

$$
\sup _{\mathbb{H}^{n} \backslash B_{r}\left(p_{0}\right)}|g(\cdot, 0)-h| \rightarrow 0 \text { as } r \rightarrow \infty
$$

and $g$ is $\varepsilon$-close to $h$, where $\varepsilon \leq \varepsilon(n)$ is sufficiently small. Then for every $0<\tau<T$ and $0<\sigma \leq 1$ there exists an $R_{0}>0$ such that

$$
\sup _{\left(\mathbb{H}^{n} \backslash B_{R_{0}}\left(p_{0}\right)\right) \times[0, \tau]}|g-h| \leq \sigma .
$$


Proof. Choose a smooth function $\eta: \mathbb{R} \rightarrow \mathbb{R}, 0 \leq \eta \leq 1$, such that $\eta \equiv 0$ on $(-\infty, 1], \eta \equiv 1$ on $[2,3], \eta \equiv 0$ on $[4, \infty)$. We can furthermore assume that $\left|\eta^{\prime \prime}\right| \leq 100$ and $\left|\eta^{\prime}\right|^{2} \leq 1000 \eta$.

Let $\rho_{p_{0}}(\cdot)$ denote the distance to the point $p_{0} \in \mathbb{H}^{n}$ with respect to the hyperbolic metric, and define the cut-off function

$$
\gamma_{p_{0}, R}:=\eta\left(\frac{\rho_{p_{0}}}{R}\right)
$$

Then we have, suppressing in the following the subscripts $p_{0}$ and $R$ :

$$
|\nabla \gamma| \leq \frac{\left|\eta^{\prime}\right|}{R}, \quad\left|\nabla^{2} \gamma\right| \leq \frac{c(n)}{R}+\frac{c(n)}{R^{2}} .
$$

Define $\psi:=\gamma|Z|^{2}$, where $Z=g-h$. Using Lemma 2.2 and the above estimates we see that

$$
\begin{aligned}
\frac{\partial}{\partial t} \psi \leq & g^{i j} \nabla_{i} \nabla_{j} \psi-2 g^{i j} \nabla_{i} \gamma \nabla_{j}|Z|^{2}-|Z|^{2} g^{i j} \nabla_{i} \nabla_{j} \gamma \\
& -(2-\varepsilon) \gamma|\nabla Z|^{2}+(4+\varepsilon) \psi \\
\leq & g^{i j} \nabla_{i} \nabla_{j} \psi+(4+\varepsilon) \psi+\frac{c(n) \varepsilon}{R}+\frac{c(n) \varepsilon}{R^{2}}
\end{aligned}
$$

where we used the estimates on the derivatives of $\gamma$ and Kato's inequality to estimate the terms appearing in the first inequality. More explicitly

$$
\left.|| Z\right|^{2} g^{i j} \nabla_{i} \nabla_{j} \gamma \mid \leq c(n) \varepsilon^{2}\left(\frac{c(n)}{R}+\frac{c(n)}{R^{2}}\right)
$$

and

$$
\begin{aligned}
-(2-\varepsilon) \gamma|\nabla Z|^{2}-2 g^{i j} \nabla_{i} \gamma \nabla_{j}|Z|^{2} \leq & -(2-\varepsilon) \gamma|\nabla Z|^{2}+c(n)|\nabla \gamma||Z||\nabla Z| \\
\leq & -(2-\varepsilon) \gamma|\nabla Z|^{2}+(c(n) / R) \sqrt{\gamma}|Z||\nabla Z| \\
\leq & -(2-\varepsilon) \gamma|\nabla Z|^{2}+\gamma|\nabla Z|^{2} \\
& +(c(n) / R)^{2}|Z|^{2} \\
\leq & \varepsilon \frac{c(n)}{R^{2}} .
\end{aligned}
$$

Now choosing $R$ big enough, we may apply the maximum principle to the differential inequality for $\psi$ and obtain the desired estimate. 
Lemma A.3. The scaled Ricci flow and the scaled Ricci harmonic map heat flow are related as follows. Assume $\varphi_{t}: \mathbb{H}^{n} \rightarrow \mathbb{H}^{n}$ solves

$$
\frac{\partial}{\partial t} \varphi_{t}(x, t)=-V\left(\varphi_{t}(x, t), t\right)
$$

where the components of $V$ are given by $V^{\alpha}:=g^{\beta \gamma}\left({ }^{g} \Gamma_{\beta \gamma}^{\alpha}-{ }^{h} \Gamma_{\beta \gamma}^{\alpha}\right)$ and that the $\varphi_{t}: \mathbb{H}^{n} \rightarrow \mathbb{H}^{n}$ are smooth and diffeomorphisms for all time. Let $\left(\mathbb{H}^{n}, g(t)\right)_{t \in[0, T)}$ be a solution to the scaled Ricci harmonic map heat flow (1.3), $V_{i}=g_{i \alpha} V^{\alpha}$. Then $\left(\mathbb{H}^{n}, \tilde{g}(t)\right)_{t \in[0, T)}$ solves the scaled Ricci flow (1.2), with $\tilde{g}(0)=g(0)$, where here $\tilde{g}(t):=\varphi_{t}^{*}(g(t))$.

Proof. For $\tilde{g}(t):=\varphi_{t}^{*} g(t)$, we get

$$
\begin{aligned}
\frac{\partial}{\partial t}(\tilde{g}(t))= & \left(\varphi_{t}\right)^{*}\left(\frac{\partial}{\partial t} g\right)+\left.\frac{\partial}{\partial s}\right|_{s=0}\left(\varphi_{t+s}^{*} g(t)\right) \\
= & -2 \operatorname{Ric}(\tilde{g}(t))-2(n-1) \tilde{g}(t)+\varphi_{t}^{*}\left(\mathcal{L}_{V(t)} g(t)\right) \\
& -\mathcal{L}_{\left(\varphi_{t}^{-1}\right)_{*} V(t)}\left(\varphi_{t}^{*} g(t)\right) \\
= & -2 \operatorname{Ric}(\tilde{g}(t))-2(n-1) \tilde{g}(t)
\end{aligned}
$$

where here $\mathcal{L}_{W} k$ is the Lie-derivative of $k$ in the direction $W$ (in coordinates $\left.\left(\mathcal{L}_{W} k\right)_{i j}={ }^{k} \nabla_{i} W_{j}+{ }^{k} \nabla_{j} W_{i}\right)$, see $[2$, Chapter 2, Section 6].

Lemma A.4. The Ricci flow

$$
\frac{\partial}{\partial t} g=-2 \operatorname{Ric}(g)
$$

and the scaled Ricci flow (1.2) are equivalent in the following sense.

Let $\left(\mathbb{H}^{n}, \tilde{g}(\tilde{t})\right)_{\tilde{t} \in[0, \tilde{T})}$ be a solution to the scaled Ricci flow. Define $\left(\mathbb{H}^{n}, g(t)\right)_{t \in[0, T)}$ by

$$
g(\cdot, t):=(1+2(n-1) t) \tilde{g}(\cdot, \tilde{t}(t))
$$

where

$$
\tilde{t}(t):=\frac{\log (1+2(n-1) t)}{2(n-1)}
$$

and $T:=\frac{\mathrm{e}^{2(n-1) \tilde{T}}-1}{2(n-1)}$. 
Then $\left(\mathbb{H}^{n}, g(t)\right)_{t \in[0, T)}$ solves the Ricci flow. Alternatively, let $\left(\mathbb{H}^{n}, g(t)\right)_{t \in[0, T)}$ be a solution to the Ricci flow. Define $\left(\mathbb{H}^{n}, \tilde{g}(\tilde{t})\right)_{\tilde{t} \in[0, \tilde{T})}$ by

$$
\tilde{g}(\cdot, \tilde{t}):=\mathrm{e}^{-2(n-1) \tilde{t}} g(\cdot, t(\tilde{t})),
$$

where $t(\tilde{t}):=\frac{\mathrm{e}^{2(n-1) \tilde{t}}-1}{2(n-1)}$ and $\tilde{T}:=\frac{\log (1+2(n-1) T)}{2(n-1)}$. Then $\tilde{g}$ solves the scaled Ricci flow.

Proof. We prove the first claim by calculating. The second claim is shown in a similar way. We calculate at $t_{0}$, and let $\tilde{t}_{0}:=\frac{\log \left(1+2(n-1) t_{0}\right)}{2(n-1)}$.

$$
\begin{aligned}
\left(\frac{\partial}{\partial t} g\right)\left(\cdot, t_{0}\right) & =2(n-1) \tilde{g}\left(\cdot, \tilde{t}_{0}\right)+\left(\frac{\partial}{\partial \tilde{t}} \tilde{g}\right)\left(\cdot, \tilde{t}_{0}\right) \\
& =2(n-1) \tilde{g}\left(\cdot, \tilde{t}_{0}\right)-2 \operatorname{Ric}\left(\tilde{g}, \tilde{t}_{0}\right)-2(n-1) \tilde{g}\left(\cdot, \tilde{t}_{0}\right) \\
& =-2 \operatorname{Ric}\left(\tilde{g}\left(\tilde{t}_{0}\right)\right) \\
& =-2 \operatorname{Ric}\left(g\left(t_{0}\right)\right)
\end{aligned}
$$

where the last line follows from the fact, that the Ricci tensor is invariant under scaling of the metric.

Lemma A.5. Let $u \in C^{2}$ on $[0, \infty)$ or $\mathbb{R}$. Then

$$
\|D u\|_{L^{\infty}}^{2} \leq 32 \cdot\|u\|_{L^{\infty}} \cdot\left\|D^{2} u\right\|_{L^{\infty}} .
$$

Proof. Assume without loss of generality that $D u(0) \geq \frac{1}{2}\|D u\|_{L^{\infty}}=: \frac{1}{2} M$. Then $D u(x) \geq \frac{1}{4} M$ for all $0 \leq x \leq \frac{M}{4 \cdot\left\|D^{2} u\right\|_{L^{\infty}}}$. Hence

$$
2\|u\|_{L^{\infty}} \geq\left|u\left(\frac{M}{4 \cdot\left\|D^{2} u\right\|_{L^{\infty}}}\right)-u(0)\right| \geq \frac{M}{4} \cdot \frac{M}{4 \cdot\left\|D^{2} u\right\|_{L^{\infty}}} .
$$

The claim follows.

Lemma A.6. Let $B$ be a compact subset of a Riemannian manifold $M$. Assume that $B$ has $C^{2}$-boundary. Let $u \in C^{2}(M)$. Then

$$
\|\nabla u\|_{L^{\infty}(B)}^{2} \leq c(B) \cdot\|u\|_{L^{\infty}(B)} \cdot\left(\left\|\nabla^{2} u\right\|_{L^{\infty}(B)}+\|\nabla u\|_{L^{\infty}(B)}\right) .
$$

Proof. For every point $p \in B$ and every unit vector $\xi \in T_{p} M$ there exists a curve $\gamma:[0, \infty) \rightarrow B$, parameterized by arc-length, such that $\gamma(0)=p$, 
$\gamma^{\prime}(0) \in \pm\{\xi\}$ and

$$
\begin{aligned}
\left|(u \circ \gamma)^{\prime}(t)\right| & \leq|\nabla u(\gamma(t))| \\
\left|(u \circ \gamma)^{\prime \prime}(t)\right| & \leq c(B) \cdot\left(\left|\nabla^{2} u(\gamma(t))\right|+|\nabla u(\gamma(t))|\right) .
\end{aligned}
$$

Note that $c(B)$ depends on the curvature of $\gamma$ but can be chosen uniformly for all $p \in B$. Choosing $p$ and $\xi$ such that $\nabla u$ attains its maximum at $p$ in direction $\xi$, Lemma A.5 yields the statement.

\section{B. Euclidean space}

We consider the situation of the main theorem, Theorem 1.3, in [8]. Instead of a Lyapunov function involving $\varphi_{m}+\psi_{m}-2 n=\sum_{i=1}^{n} \frac{1}{\lambda_{i}^{m}}\left(\lambda_{i}^{m}-1\right)^{2}$, however, we study a Lyapunov function involving $|g-h|^{p}, p \geq 2$. This simplifies the proof.

Recall that the Ricci harmonic map heat flow with Euclidean background metric is

$$
\begin{aligned}
\frac{\partial}{\partial t} g_{i j}= & g^{a b} \nabla_{a} \nabla_{b} g_{i j}+\frac{1}{2} g^{a b} g^{p q}\left(\nabla_{i} g_{p a} \nabla_{j} g_{q b}+2 \nabla_{a} g_{j p} \nabla_{q} g_{i b}\right. \\
& \left.-2 \nabla_{a} g_{j p} \nabla_{b} g_{i q}-2 \nabla_{j} g_{p a} \nabla_{b} g_{i q}-2 \nabla_{i} g_{p a} \nabla_{b} g_{j q}\right)
\end{aligned}
$$

where $\nabla$ denotes covariant differentiation w.r.t. the Euclidean metric $h$. Calculating as in Lemma 2.2, we see that

$$
\frac{\partial}{\partial t}|g-h|^{2}-g^{i j} \nabla_{i} \nabla_{j}|g-h|^{2} \leq-\left(\frac{2}{1+\varepsilon}-9 \varepsilon(1+\varepsilon)^{2}\right)|\nabla g|^{2} \leq 0
$$

if $0<\varepsilon \leq \frac{1}{7}$. Note that there is no zeroth-order term in the evolution equation on Euclidean space. Hence $|g(t)-h| \leq \varepsilon$ is preserved during the flow and we obtain long-time existence, see [10]. Define

$$
I_{\delta}^{p}(t):=\int_{\mathbb{R}^{n}}\left(|g-h|^{p}-\delta\right)_{+} .
$$

Using (B.1), and calculating as in [8], we get

$$
\frac{d}{d t} I_{\delta}^{p}(t) \leq-\int_{\left\{|g-h|^{p}>\delta\right\}} \frac{p}{2} \cdot \frac{2-(11+9 \varepsilon)(1+\varepsilon)^{2} \varepsilon}{1+\varepsilon} \cdot|g-h|^{p-2} \cdot|\nabla g|^{2} \leq 0
$$

for $0<\varepsilon \leq \frac{1}{8}$. The rest of the proof is similar to [8]. If we further restrict $p$ to $2 \leq p<n$ then we can argue as in the paper [8] to prove Theorem 1.4 of that paper. 


\section{Conformal Ricci flow in two dimensions}

Let us consider the Euclidean ball $B:=B_{1}(0) \subset \mathbb{R}^{2}$ equipped with the metric $\left(g_{i j}\right)=\left(\mathrm{e}^{f+u} \delta_{i j}\right)$, where $f=\log 4-2 \log \left(1-|x|^{2}\right)$ and $u=u(x, t)$. For $u \equiv 0$, we get hyperbolic space of sectional curvature equal to -1 . Consider rescaled Ricci flow

$$
\frac{\partial}{\partial t} g_{i j}=-2 R_{i j}-2 g_{i j}
$$

As $R_{i j}=-\frac{1}{2} \delta_{i j} \Delta_{\delta}(u+f)$, this is equivalent to

$\dot{u}=\mathrm{e}^{-u-f} \Delta_{\delta} u+2\left(\mathrm{e}^{-u}-1\right)=\mathrm{e}^{-u} \Delta_{h} u+2\left(\mathrm{e}^{-u}-1\right)=\Delta_{g} u+2\left(\mathrm{e}^{-u}-1\right)$.

In contrast to Theorem 1.3, we do not have to assume that the eigenvalues $\left(\lambda_{i}\right)$ of $g(0)$ with respect to $h$ are close to one. This is similar to [8, Theorem A.1]. There, however, we had to assume that $\lambda_{i}(x, 0) \rightarrow 1$ for $|x| \rightarrow \infty$ in order to obtain convergence to $\mathbb{R}^{2}$, see $[8$, Th. A.2].

Theorem C.1. Let $u_{0} \in C^{0}(B)$ satisfy $\left\|u_{0}\right\|_{L^{\infty}}<\infty$. Then there exists $u \in$ $C^{\infty}(B \times(0, \infty))$ solving $(\mathrm{C} .1)$ such that $u(\cdot, t) \rightarrow u_{0}$ in $C_{l o c}^{0}(B)$ as $t \searrow 0$. As $t \rightarrow \infty, u(\cdot, t) \rightarrow 0$ exponentially in $C^{\infty}$ w.r.t. the hyperbolic metric.

If an arbitrary solution $u$ is uniformly bounded for small times, we also get exponential convergence.

Proof. Assume $\left|u_{0}\right| \leq c_{0}$. Mollify and modify $u_{0}$ to $u_{0}^{i}$ with $\left|u_{0}^{i}\right| \leq 2 c_{0}$ and $u_{0}^{i}=0$ near $\partial B_{1-\frac{1}{i}}(0)$ and $u_{0}^{i}=u_{0}$ on $B_{1-\frac{2}{i}}(0)$. We can construct solutions $u^{i}: B_{1-\frac{1}{i}}(0) \times\left[0, T_{i}\right)$ to C. 1 with $u^{i}(\cdot, 0)=u_{0}$ on $B_{1-\frac{1}{i}}(0)$ and $u_{i}(\cdot, t)=0$ on $\partial B_{1-\frac{1}{i}}(0)$ using the arguments presented in Chapter VI of [5]. These solutions remain bounded by $2 c_{0}$ from the maximum principle. Hence, the arguments of Chapter VI of [5] imply that $T_{i}=\infty$.

Spatially constant barriers $b=b(t)=\log \left(1+a \mathrm{e}^{-2 t}\right), a>-1$, solving (C.1) converge exponentially to 0 as $t \rightarrow \infty$. Hence the maximum principle applied to each $u^{i}$ on $B_{1-\frac{1}{i}}(0)$ implies that the $u_{i}$ remain uniformly bounded and approach zero exponentially (uniformly in $i$ ) as $t \rightarrow \infty$.

Now we address smooth convergence: Writing $l^{i}:=\mathrm{e}^{u^{i}}$ we obtain the evolution equation

$$
\frac{\partial}{\partial t} l^{i}=\left(1 / l^{i}\right) \Delta_{h} l^{i}-\left.\left.\left(1 /\left(l^{i}\right)^{2}\right)\right|^{h} \nabla l^{i}\right|^{2}+2\left(1-l^{i}\right) .
$$

We can assume without loss of generality, that $\left|l_{i}-1\right| \leq \varepsilon$ for some small $\varepsilon$. The interior estimates of Lemma A.1 hold here as the equation for $l^{i}$ has 
the same form as the equation studied in Theorem 4.3 of [10]. Hence, by taking a diagonal subsequence, we get a solution $l=\mathrm{e}^{u}$ which approaches 1 exponentially. Interpolating between the $C^{0}$-norm and $C^{k}$-norms and using Lemma A.1 again, we see that $l$ approaches 1 in all $C^{k}$-norms exponentially.

To treat the question of uniqueness of such solutions we work in the unrescaled setting. Note that by Lemma A.4 this is equivalent to the rescaled equation.

With respect to the hyperbolic metric $h$ on $\mathbb{H}^{2}$ as a background metric, a solution $\mathrm{e}^{u(p, t)} h$ to the Ricci flow satisfies

$$
\dot{u}=\mathrm{e}^{-u} \Delta_{h} u+2 \mathrm{e}^{-u} .
$$

We first prove a non-compact maximum principle.

Lemma C.1. Let $v \in C^{\infty}\left(\mathbb{H}^{2},[0, T)\right)$ be a bounded solution to

$$
\dot{v} \leq a \Delta_{h} v+c v
$$

with $a, c \in L^{\infty}\left(\mathbb{H}^{2},[0, T)\right), a>0$. If $v(\cdot, 0) \leq 0$ then $v(\cdot, t) \leq 0$ for all $t \in$ $[0, T)$.

Proof. Pick a fixed point $p_{0} \in \mathbb{H}^{2}$ and let $r(\cdot):=\operatorname{dist}_{h}\left(\cdot, p_{0}\right)$. Then the function $\rho:=\sqrt{r^{2}+1}$ is a smooth function on $\mathbb{H}^{2}$ with $\rho(p) \rightarrow \infty$ as $p \rightarrow \infty$ and

$$
a \Delta_{h} \rho \leq C
$$

for a constant $C>0$. Let us first assume that $v$ satisfies

$$
\dot{v} \leq a \Delta_{h} v-c^{\prime} v
$$

with a function $c^{\prime} \geq 0$. Then for any $\delta>0$ the function

$$
w:=v-\delta \rho-2 \delta C t-\delta
$$

satisfies at the first non-negative interior maximum

$$
\dot{w}<a \Delta_{h} w .
$$

Since $w(\cdot, t) \rightarrow-\infty$ as $p \rightarrow \infty$ an application of the maximum principle proves the estimate as $\delta \rightarrow 0$. In the general case let $|c(p, t)| \leq K$ and $v^{\prime}:=$ 
$\mathrm{e}^{-K t} v$ which satisfies

$$
\dot{v}^{\prime} \leq a \Delta_{h} v^{\prime}-(K-c) v^{\prime}
$$

The previous estimate can be applied.

This gives us a uniqueness statement.

Lemma C.2. Let $u, \tilde{u} \in C^{\infty}\left(\mathbb{H}^{2},(0, T)\right) \cap C^{0}\left(\mathbb{H}^{2},[0, T)\right)$ be two bounded solutions of (C.2) s.t.

$$
u(\cdot, t) \rightarrow u_{0} \text { and } \tilde{u}(\cdot, t) \rightarrow u_{0}
$$

uniformly as $t \rightarrow 0$ for some continuous function $u_{0}$ on $\mathbb{H}^{2}$. Then $u \equiv \tilde{u}$.

Proof. Define for $\gamma>0$

$$
u_{\gamma}(p, t):=u\left(p, \mathrm{e}^{-\gamma} t\right)+\gamma
$$

Then $u_{\gamma}$ again solves (C.2) with initial values $u_{0}+\gamma$. Since the initial values are attained uniformly we have $u_{\gamma}>\tilde{u}$ for a short-time interval $[0,2 \delta], \delta>0$. By interior estimates as in Lemma A.1 the functions $u_{\gamma}, \tilde{u}$ are bounded uniformly in $C^{\infty}$ on time intervals $[\delta, T)$. Interpolating between the two solutions, we see that the difference satisfies an equation of the form (C.3), to which the non-compact maximum principle applies. Thus $u_{\gamma}>\tilde{u}$ for all $\gamma>0$ and $\gamma \rightarrow 0$ gives the desired estimate.

If $u_{0}$ is uniformly continuous then also $u(\cdot, t)$ converges uniformly as $t \rightarrow 0$.

Lemma C.3. Let $u \in C^{\infty}\left(\mathbb{H}^{2},(0, T)\right) \cap C^{0}\left(\mathbb{H}^{2},[0, T)\right)$ be a bounded solution of $(\mathrm{C} .2)$ s.t. $u_{0}:=u(\cdot, 0)$ is uniformly continuous. Then $u(\cdot, t) \rightarrow u_{0}$ uniformly.

Proof. Pick a point $p_{0} \in \mathbb{H}^{2}$ and let $u_{p_{0}}(t)$ be the solution to (C.2), which is constant in space and has initial value $u\left(p_{0}\right)$. It is a direct computation to check that

$$
\begin{aligned}
\frac{\partial}{\partial t}\left(u-u_{p_{0}}\right)^{2} & =\mathrm{e}^{-u} \Delta_{h}\left(u-u_{p_{0}}\right)^{2}-2 \mathrm{e}^{-u}\left|\nabla^{h} u\right|^{2}+2\left(u-u_{p_{0}}\right)\left(\mathrm{e}^{-u}-\mathrm{e}^{-u_{p_{0}}}\right) \\
& \leq \mathrm{e}^{-u} \Delta_{h}\left(u-u_{p_{0}}\right)^{2}-2 \mathrm{e}^{-u}\left|\nabla^{h} u\right|^{2}
\end{aligned}
$$

By a similar argument as in Lemma A.2, but now for small radii, one obtains the desired closeness-estimate. 
Corollary C.1. Any bounded solution to (C.1) in $C^{\infty}\left(\mathbb{H}^{2},(0, \infty)\right) \cap C^{0}$ $\left(\mathbb{H}^{2},[0, T)\right)$ with initial data $u_{0}$, which is uniformly continuous with respect to the hyperbolic metric, is unique. As $t \rightarrow \infty, u(\cdot, t) \rightarrow 0$ exponentially in $C^{\infty}$.

\section{Acknowledgment}

O.C.S. and F.S. were partially supported by SFB 647, M.S. by SFB/ Transregio 71.

\section{References}

1. R. Bamler, Stability of symmetric spaces of noncompact type under Ricci flow, arXiv:math.DG/1011.4267v1.

2. B. Chow, P. Lu and L. Ni, Hamilton's Ricci flow, in 'Graduate Studies in Mathematics', 77, American Mathematical Society, Providence, RI, 2006.

3. D.M. DeTurck, Deforming metrics in the direction of their Ricci tensors, J. Diff. Geom. 18(1) (1983), 157-162.

4. R.S. Hamilton, Three-manifolds with positive Ricci curvature, J. Diff. Geom. 17(2) (1982), 255-306.

5. O.A. Ladyženskaja, V.A. Solonnikov and N.N. Ural'ceva, Linear and quasilinear equations of parabolic type, Translated from the Russian by S. Smith. Translations of Mathematical Monogr., 23, American Mathematical Society, Providence, RI, 1967.

6. H. Li and H. Yin, On stability of the hyperbolic space form under the normalized Ricci flow, Int. Math. Res. Not. IMRN (2010), doi : 10.1093/imrn/rnp232.

7. H.P. McKean, An upper bound to the spectrum of $\Delta$ on a manifold of negative curvature, J. Diff. Geom. 4 (1970), 359-366.

8. O.C. Schnürer, F. Schulze and M. Simon, Stability of Euclidean space under Ricci flow, Comm. Anal. Geom. 16(1) (2008), 127-158.

9. W-X Shi, Deforming the metric on complete Riemannian manifolds, J. Diff. Geom. 30(1) (1989), 223-301. 
10. M. Simon, Deformation of $C^{0}$ Riemannian metrics in the direction of their Ricci curvature, Comm. Anal. Geom. 10(5) (2002), 1033-1074.

11. V. Suneeta, Investigating the off-shell stability of anti-de Sitter space in string theory, Class. Quant. Grav. 26(3) (2009), 035023, 18.

12. R. Ye, Ricci flow, Einstein metrics and space forms, Trans. Amer. Math. Soc. 338(2) (1993), 871-896.

Fachbereich Mathematik und Statistik

UNIVERSITÄT KONSTANZ

78457 KONSTANZ

GERMANY

E-mail address: Oliver.Schnuerer@uni-konstanz.de

Freie Universität BerLin

Arnimallee 6

14195 BERLIN

Germany

E-mail address: Felix.Schulze@math.fu-berlin.de

INSTITUt FÜR ANALYSIS UND NUMERIK (IAN)

UNIVERSITÄTSPLATZ 2, G02-19A

39106 MAGDEBURG

Germany

E-mail address: msimon@gmx.de

Received March 19, 2010 
